\title{
Development, Reproductive Output and Population Growth of the Fruit Fly Pest Drosophila suzukii (Diptera: Drosophilidae) on Artificial Diet
}

\author{
LISA M. EMILJANOWICZ, GERALDINE D. RYAN, ${ }^{1}$ AARON LANGILLE, AND JONATHAN NEWMAN \\ School of Environmental Sciences, University of Guelph, Guelph, Ontario N1G 2M7 Canada
}

\begin{abstract}
J. Econ. Entomol. 107(4): 1392-1398 (2014); DOI: http://dx.doi.org/10.1603/EC13504
ABSTRACT Drosophila suzukii (Matsumura) (Diptera: Drosophilidae) is a fruit pest of Asian origin that invaded North America in 2008. Despite the widespread economic impact of this species, much of the biology and general life history of this pest remains largely unknown. Under optimal laboratory conditions $\left(22^{\circ} \mathrm{C}, \approx 25 \%\right.$ relative humidity), we measured development, survival, fecundity, hatch rate, and sex ratio of a North American ecotype of D. suzukii. Life history traits were used to construct a life table and reproductive schedule, and to calculate the intrinsic rate of population increase. The mean $( \pm$ SE) total lifespan (egg to adult mortality) was $86.1 \pm 4.25 \mathrm{~d}$, with a maximum value of $153.7 \mathrm{~d}$. On average, females produced $5.7 \pm 0.24$ eggs per day, with a mean total lifetime production of 635.6 eggs. The gross reproductive rate was 317.8 daughter eggs per female and the net reproductive rate was 240.4 daughter eggs per female. The intrinsic rate of natural increase was 0.179 . The stable age distribution $\left(c_{*}\right)$ was comprised of $51 \%$ larvae, $25 \%$ eggs, $16 \%$ pupae, and $8 \%$ adults. The sex ratio over time was $\approx 1: 1$. We conclude with a comparison of our data with previous work on $D$. suzukii and other Drosophila, and discuss the implications for control and monitoring of this pest.
\end{abstract}

KEY WORDS development, Drosophila suzukii, intrinsic rate of increase, life history, spotted wing drosophila

Drosophila suzukii (Matsumura) (Diptera: Drosophilidae), commonly called spotted wing drosophila, is an invasive fruit fly native to Southeast Asia, and an opportunistic pest of a wide range of soft-skinned fruit species (Bolda et al. 2010, Lee et al. 2011b). D. suzukii is able to lay eggs in ripening fruit, using their serrated ovipositor, before harvest has occurred, resulting in yield loss (Lee et al. 2011b, Walsh et al. 2011). They have recently invaded the United States, Canada, and Europe. The first North American mainland detection of D. suzukii was in California in August 2008 and the first European detection was in Spain (later Italy and France) in October 2008 (Walsh et al. 2011, Hauser 2011). The following year the infestation spread, resulting in fruit crop damage and revenue loss (Hauser 2011). D. suzukii continued to spread along the west coast of North America and was identified in British Columbia in 2009, and subsequently in most fruitproducing locations of Canada as of 2010 (Canadian Food Inspection Agency [CFIA] 2011).

General life history measurements on D. suzukii were originally conducted by Kanzawa (1939) in Japan. Kanzawa found that females reach sexual maturity 1-2 d after emerging, producing between 219 and 563 eggs over their lifetime, with full development (egg to adult) taking $\approx 14.6 \mathrm{~d}$ to complete (Lee et al.

${ }^{1}$ Corresponding author, e-mail: gryan@uoguelph.ca. 2011b, Walsh et al. 2011). Although such life history measures are critical for modeling the full impact of this fly, these early measurements may not accurately capture the life history of the North American ecotype $>70$ yr later. For example, in Drosophila melanogaster (Meigen) it has been found that artificial laboratory selection for early fecundity can result in a significant change over a short period of time (Rose and Charlesworth 1980) and that fecundity and fertility show evidence of adaptation to certain temperature regimes (Partridge et al. 1995). There is also evidence suggesting evolutionary change in natural populations of Drosophila, including changes in chromosomal polymorphisms in response to environmental change (Levitan and Etges 2005) and wing morphological evolution in response to latitude (Huey et al. 2000). Given this evolutionary capacity of Drosophila, differences may be present between the previously studied Asian population and the recently introduced North American populations of $D$. suzukii. It is also likely that upon invasion, the North American population experienced a genetic bottleneck, where genetic variation is greatly reduced in a founding population. This has been reported previously for an introduced population of an invasive insect pest, the glassy-winged sharpshooter (Stenger et al. 2010). Therefore, life history exploration is important to understand the potential of this pest in North America. 
Life history and reproductive measures can highlight the fitness potential of organisms and their study can be useful when dealing with pests, such as $D$. suzukii and Cimex lectularius L. (Partridge et al. 1995, Polanco et al. 2011). As D. suzukii is a fairly new pest to North American growers, minimal pest management work has been conducted to understand the best preventative measures. Preliminary work by Beers et al. (2011) suggest that some pesticides may provide suitable protection against $D$. suzukii activity; however, the main issue that warrants resolution is time of management. By understanding the phenology, reproduction, and growth potential of D. suzukii, management of this pest may be improved.

In the current study, we measured various D. suzukii life history and reproductive parameters under optimal laboratory conditions. These data were then used to construct an abridged life table, reproductive schedule, and to calculate the intrinsic rate of natural increase $(r)$ and stable age distribution.

\section{Methods}

D. suzukii Rearing and Colony Maintenance. All insects used were obtained from a laboratory colony that originated from infested fruit collected from a commercial blackberry and raspberry farm in southern Ontario during the summer of $2012(\approx 8$ mo before life history calculations). Positively identified $D$. suzukii were then kept in cages (Plexiglas 26 by 26 by 26 $\mathrm{cm})$ with overlapping generations on standard Drosophila diet (as is described in Dalton et al. 2011) and cotton saturated with double-distilled water. The $D$. suzukii colonies were kept in controlled growth chambers at $22 \pm 0.00089^{\circ} \mathrm{C}\left(\right.$ ranging $\left.21.45-22.53^{\circ} \mathrm{C}\right), \approx 25 \%$ relative humidity $(\mathrm{RH})$, and a photoperiod of 15:9 (L:D) h. To maintain the colonies, petri dishes (Fisherbrand 100 by $25 \mathrm{~mm}$, Fisher, Ottawa, ON, Canada) filled with artificial diet were left in the cage so that females could oviposit eggs into the diet. Dishes were removed after $\approx 4 \mathrm{~d}$ and left to incubate in the same conditions until adults had emerged. These adults were then reintroduced back into the same cage.

Fecundity, Hatch Rate, and Sex Ratio Measurements. To conduct fecundity measures on individual females, mating chambers were constructed from modified centrifuge tubes (Fisherbrand $50 \mathrm{ml}$, Fisher, Ottawa, ON, Canada). A hole was cut in the side of the tube and a small section of clear PVC tubing was glued inside the hole where cotton could be inserted and saturated with double-distilled water. This cotton was rehydrated with double-distilled water every $3 \mathrm{~d}$ during the study period. A second hole was cut into the opposite side of the centrifuge tube and covered with mesh. Although these mating chambers were kept within the controlled growth chamber, it is possible that the $\mathrm{RH}$ was slightly higher than $25 \%$ within the mating chamber.

Adults used in the study to calculate reproductive measures were reared from the laboratory colony by removing a pupa from a day old diet dish and placing it into a microcentrifuge tube (Fisherbrand $1.5 \mathrm{ml}$,
Fisher, Ottawa, ON, Canada) half-filled with standard diet medium. Once these adults had emerged, they were sexed and placed into a mating chamber (two males and one female into each chamber to ensure mating success). In total, 50 mating chambers were set up in this way. A subset of 25 chambers contained the same males throughout the study (referred to as "nonreplacement male" group), while the males in the other 25 chambers were replaced weekly with newly emerged, virgin males (referred to as "replacement male" group). This was done to test whether our measurements of the female reproductive period were affected by the length of the male reproductive period. Thus, we hypothesized that if the male reproductive period was shorter than the female period, we would observe lower hatch rate of eggs from the nonreplacement male group.

Egg counts were conducted daily on all 50 chambers. The lids of the centrifuge tubes were filled with $1 \mathrm{ml}$ of diet upon which females would deposit eggs. Lids were replaced daily and eggs were counted. After egg laying, these lids were left for $\approx 4 \mathrm{~d}$ to allow for larval counts. This provided a hatch rate measure for the eggs. These counts were made until the female in the chamber died. Males that died in the nonreplacement male group were not replaced. Males that had died in the replacement male group were replaced on the regular replacement schedule.

To determine sex ratio, the diet from the hatch rate lids of the nonreplacement male group were transferred to a petri dish (Fisherbrand 35 by $10 \mathrm{~mm}$, Fisher, Ottawa, ON, Canada) filled with diet, and left to develop into adults. Once adults had emerged, the sex ratio was assessed. This was done starting $1 \mathrm{wk}$ into the study, and was repeated 3 times a week on alternate weeks throughout the study period. We stopped measuring sex ratio after $87 \mathrm{~d}$ (13 time points) when our sample size became reduced because of female mortality.

D. suzukii Survivorship and Development Measurements. To determine development time and survivorship for each life stage, we checked the nonreplacement male mating chambers every $2 \mathrm{~h}$ for egg production over a 3 -d period, $\approx 10 \mathrm{~d}$ posteclosion. We collected up to 3 eggs from each chamber to obtain a cohort of 53 eggs to track our measures of interest. Individual eggs were obtained by scooping a small amount of diet around the egg with a pair of forceps and transferring the mass to a petri dish (Fisherbrand 35 by $10 \mathrm{~mm}$, Fisher, Ottawa, ON, Canada) filled with diet. Eggs were checked every $2 \mathrm{~h}$ until they had hatched into larvae. If an egg did not hatch after $3 \mathrm{~d}$, it was considered a nonviable egg. Larvae were checked every $4 \mathrm{~h}$, through the three instars, until they had pupated. Pupae were checked every $8 \mathrm{~h}$ until adults had emerged. The midpoint of these intervals was used when calculating the development time in each stage. As we could not sex immature individuals (because of lack of morphological identifiers; Cini et al. 2012), mortality at these stages (egg, larvae, and pupae) was assumed to be equal for both males and females. Individual adults were then sexed and trans- 
Table 1. Life table and reproductive schedule parameters used as defined by Carey $(1982,1993)$

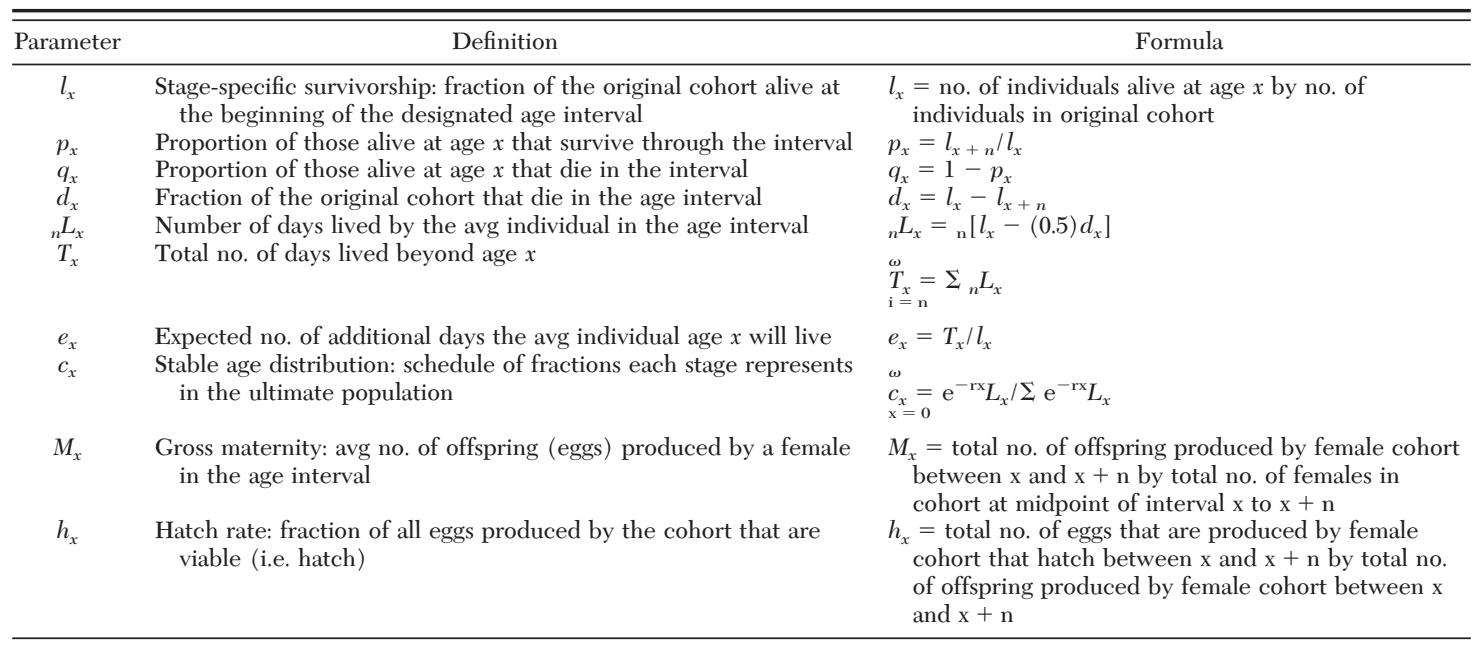

ferred singly to a mating chamber, and checked daily to measure survivorship. The diet lid was replaced and the cotton was rehydrated with double-distilled water every $3 \mathrm{~d}$.

Life Tables and Data Analysis. Survival data were used to construct an abridged life table, containing stage-specific measures as opposed to daily measures, by assessing mortality over an age interval $(n)$. The adult stage was grouped into intervals of $10 \mathrm{~d}$. An abridged life table was chosen, as it is difficult to determine daily mortality of immature stages. The life table included all of the stage-specific parameters shown in Table 1 . In addition, life table entropy $(H)$, a measure of heterogeneity in the probability of dying at each age (Carey 1993), was calculated.

The definitions of reproductive variables and notation used are summarized in Table 2. Fecundity data were used to construct an abridged reproductive schedule for the nonreplacement and replacement male groups. The reproductive schedule includes measures of average number of days lived in each interval $\left({ }_{n} L_{x}\right)$, gross maternity $\left(M_{x}\right)$, and hatch rate $\left(h_{x}\right)$. The formulae used for these parameters can also be found in Table 1 . The average number of eggs produced by a female in the age interval $\left(M_{x}\right)$ was calculated for the female cohort. These values were then used to calculate the parameters shown in Table 2 . The $r$ was first estimated using two analytical approximations (Carey 1993). The values obtained from these approximations were averaged to yield a value of 0.125 , and this was then used as the net reproductive rate value in the iterative method, where three iterations were carried out to arrive at a final estimate of $r$. Sex ratio was also calculated (males per female).

We determined the average time in each developmental stage, as well as total lifespan (egg to adult mortality). All statistical analyses were performed using JMP 11 (JMP 11, SAS Institute 2013, Cary, NC). A pooled variance $t$-test was used to analyze the lifespan data for males and females to test for a difference

Table 2. Variables and notation used throughout as defined by Carey $(1982,1993)$, and the measures for D. suzukii

\begin{tabular}{|c|c|c|}
\hline Notation & Definition & Estimate \\
\hline Gross fecundity rate & $\begin{array}{l}\text { Lifetime production of offspring (eggs) by an avg female that lives } \\
\text { to the last day of life in the cohort }\end{array}$ & 635.6 eggs \\
\hline Gross fertility rate & $\begin{array}{l}\text { Lifetime production of viable eggs by an avg female that lives to the } \\
\text { last day of life in the cohort }\end{array}$ & 491.1 fertile eggs \\
\hline Gross hatch rate & $\begin{array}{l}\text { Ratio of gross fertility to gross fecundity (weighs hatch by the no. of } \\
\text { eggs produced at each age) }\end{array}$ & 0.77 \\
\hline Net fecundity rate & Average lifetime production of eggs for a newborn female & 480.7 eggs \\
\hline Net fertility rate & Average lifetime production of viable eggs for a newborn female & 386.8 fertile eggs \\
\hline Gross reproductive rate & Sum of all female offspring per female across all ages & $\begin{array}{l}317.8 \text { daughter eggs } \\
\text { per female }\end{array}$ \\
\hline Net reproductive rate & $\begin{array}{l}\text { Average no. of female offspring that would be born to a birth cohort } \\
\text { of females during their lifetime }\end{array}$ & $\begin{array}{l}240.4 \text { daughter eggs } \\
\text { per female }\end{array}$ \\
\hline $\begin{array}{l}\text { Intrinsic rate of natural } \\
\text { increase }(r)\end{array}$ & $\begin{array}{l}\text { Rate of natural increase in a closed population that has been subject } \\
\text { to constant age-specific schedules of fertility and mortality }\end{array}$ & 0.179 \\
\hline Mean generation time & $\begin{array}{l}\text { Time required for a population to increase by a factor equal to the } \\
\text { net reproductive rate }\end{array}$ & $30.6 \mathrm{~d}$ \\
\hline Doubling time & Time required for the population to double & $3.872 \mathrm{~d}$ \\
\hline Finite rate of increase & Fraction of increase per female per unit time & 1.196 \\
\hline Life table entropy $(H)$ & Distribution of mortality by age & 0.683 \\
\hline
\end{tabular}


between the means of these groups. The $M_{x}$ and $h_{x}$ data were also analyzed by a pooled variance $t$-test to determine whether the nonreplacement male group and replacement male group differed significantly in their means. The $h_{x}$ data were arcsine square roottransformed before analysis.

\section{Results}

D. suzukii Fecundity, Hatch Rate, and Sex Ratio. The mean daily egg production across both male groups and all ages was $5.7 \pm 0.24$. All other reproductive and growth estimates are shown in Table 2. These values suggest that the lack of hatch reduced fecundity by $23 \%$, female mortality reduced fecundity by $24 \%$, and when both are taken into consideration, fecundity suffers $39 \%$ reduction. Fig. 1 shows the $M_{x}$ schedule by age for the nonreplacement and replacement male groups and Table 3 shows the abridged reproductive schedule for the nonreplacement and replacement male groups, including survival, gross maternity, and hatch rate.

The gross maternity data were found to be significantly different between the nonreplacement male group and replacement male group; the nonreplacement male group had a higher mean gross maternity $(5.9 \pm 0.17$ vs. $5.0 \pm 0.19 ; t=3.67 ; \mathrm{df}=226 ; P<0.0003)$. We did find a significant difference between the nonreplacement male group and replacement male group in the hatch rate; the replacement male group had a higher mean hatch rate $(84 \pm 0.01 \%$ vs. $71 \pm 0.02 \%$; $t=$ $-5.62 ; \mathrm{df}=221 ; P<0.0001)$.

The sex ratio of newly emerging adults remained fairly constant over the 13 time points that we considered in our study, with a mean of 0.98 males per female.

D. Suzukii Survivorship. An abridged life table for D. suzukii is presented in Table 4. An interesting result from the life table is the $e_{x}$ term (life expectancy at age $x$ ). High prepupal mortality means that a newly laid egg is expected to live for $56 \mathrm{~d}$, but by the time an individual has reached the pupal stage it has a life expectancy of $75 \mathrm{~d}$ (Table 4). After this stage, life expectancy gradually decreases until aged $112 \mathrm{~d}$ old. The brief rise in life expectancy after day 112 is probably an artifact of small sample sizes as there were only four females still living at that point. Also, when adults emerge, there is no mortality until $\approx 30 \mathrm{~d}$ of adult life. Egg to adult survivorship was $64 \%$. The stable age distribution reveals that the stage composition exists as $25 \%$ eggs, $51 \%$ larvae, $16 \%$ pupae, and $8 \%$ adults.

D. suzukii Development. Table 5 shows the mean $( \pm \mathrm{SE})$ of D. suzukii development time in days for immature stages. Total lifespan (egg to adult mortality) has a mean of $86.1 \pm 4.25 \mathrm{~d}$, with the maximum lifespan reaching $153.7 \mathrm{~d}$ for one male. The average lifespan for females was $79.5 \pm 4.86 \mathrm{~d}$, and the average lifespan for males was $93.6 \pm 6.88 \mathrm{~d}$, but these were not significantly different $(t=1.71 ; \mathrm{df}=32$; $P=0.10)$.
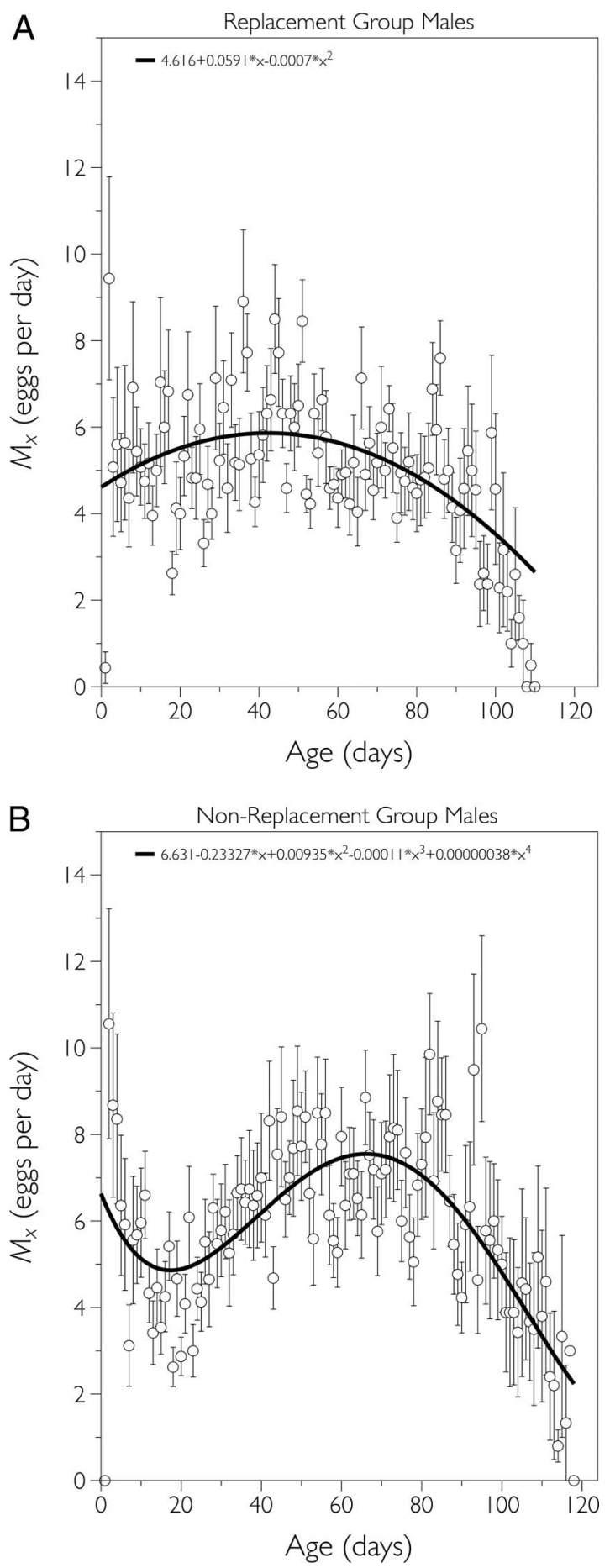

Fig. 1. The average number of eggs produced per female daily $\left(M_{x}\right)$ for the nonreplacement and replacement male groups of D. suzukii. Model selection using weighted polynomial regression and Akaike information criterion as the selection criterion suggests that the best-fit curve is the quartic for the nonreplacement male group $\left(R^{2}=0.30\right)$, and quadratic for the replacement male group $\left(R^{2}=0.42\right)$. 
Table 3. Abridged reproduction schedule for D. suzukii

\begin{tabular}{|c|c|c|c|c|}
\hline \multirow{3}{*}{$\begin{array}{c}\text { Age } \\
\text { interval } \\
1-10\end{array}$} & \multirow{2}{*}{\multicolumn{2}{|c|}{$\begin{array}{c}\begin{array}{c}\text { Nonreplacement } \\
\text { male group }\end{array} \\
l_{x} h_{x}\end{array}$}} & \multirow{2}{*}{\multicolumn{2}{|c|}{$\begin{array}{c}\begin{array}{c}\text { Replacement male } \\
\text { group }\end{array} \\
l_{x} h_{x}\end{array}$}} \\
\hline & & & & \\
\hline & 9.990 & 0.711 & 9.960 & 0.778 \\
\hline $11-20$ & 9.530 & 0.856 & 9.600 & 0.841 \\
\hline $21-30$ & 9.200 & 0.832 & 9.000 & 0.878 \\
\hline $31-40$ & 9.040 & 0.820 & 8.800 & 0.859 \\
\hline $41-50$ & 8.800 & 0.805 & 8.800 & 0.829 \\
\hline $51-60$ & 8.800 & 0.849 & 8.800 & 0.830 \\
\hline $61-70$ & 8.430 & 0.806 & 8.800 & 0.871 \\
\hline $71-80$ & 7.350 & 0.677 & 8.120 & 0.866 \\
\hline $81-90$ & 5.290 & 0.703 & 6.190 & 0.906 \\
\hline $91-100$ & 3.930 & 0.736 & 3.560 & 0.819 \\
\hline $101-110$ & 2.680 & 0.385 & 1.450 & 0.771 \\
\hline $111-120$ & 0.920 & 0.216 & 0.000 & \\
\hline
\end{tabular}

All formulae were taken from Carey $(1982,1993)$.

\section{Discussion}

To date, these data represent first reports on the life history and lifetime reproductive measures of $D$. suzukii. Baseline information on fecundity, longevity, and population growth at optimal laboratory conditions on Drosophila media is provided. Data from the current study represent a valuable benchmark as baseline information that can be used in modeling, control strategies, and to compare our population of D. suzukii with others, and other Drosophila.

Kanzawa previously reported that the average lifetime egg production of female $D$. suzukii can range from 219 to 563 eggs on cherries (as cited in Lee et al. $2011 b$ ). Our estimates were somewhat higher, where gross fecundity rate was 635.6 eggs, with lifetime egg production ranging from 92 to 868 eggs on Drosophila media. Even though it is the larvae that feed and cause deterioration of the host, it is also important to consider those eggs that do not hatch, as well as the female
Table 5. Average time (days \pm SE) in each developmental stage for the full development cohort of $D$. suzukii

\begin{tabular}{lc}
\hline \multicolumn{1}{c}{ Life stage } & Time in stage $($ days \pm SE) \\
\hline Egg $(n=44)$ & $1.4 \pm 0.08$ \\
First instar $(n=37)$ & $1.1 \pm 0.08$ \\
Second instar $(n=35)$ & $1.5 \pm 0.1$ \\
Third instar $(n=34)$ & $3.1 \pm 0.1$ \\
Total larval development $(n=33)$ & $6.0 \pm 0.2$ \\
Pupa $(n=33)$ & $5.8 \pm 0.05$ \\
Egg-adult $(n=33)$ & $12.8 \pm 0.2$ \\
\hline
\end{tabular}

$n$ is the number of individuals in each stage.

lifespan, to get a gauge of future population size. We found that the actual number of eggs that would reach adult eclosion per female in a $D$. suzukii population averages 386.8. These values of lifetime reproduction are mid-range compared with other species in the melanogaster species group, of which D. suzukii is a member. For example, Drosophila simulans (Sturtevant) have a lifetime net production of offspring that can range from 17 to 493 (Taylor et al. 2008), while $D$. melanogaster has a potential lifetime production of $>1,000$ eggs (McMillan et al. 1969). Clearly, there is heterogeneity with respect to this value within the species group.

The gross fecundity rate that we have reported could very well be a product of increased longevity. In Kanzawa's study, the adult life span was found to range from 20 to $52 \mathrm{~d}$ (Kanzawa 1939), while we found total lifespan to range from 50 to $154 \mathrm{~d}$, with a mean of $86 \pm$ $4.25 \mathrm{~d}$. Kanzawa also tracked time in each developmental stage in captivity on cherries, documenting time in the egg, larval, and pupal stages as roughly 1.4 , 7.1 , and $6.1 \mathrm{~d}$, respectively (as cited in Walsh et al. 2011). These estimates are similar to those in the current study. Previous work on D. suzukii has explored host potential and reported differences in de-

Table 4. Abridged life table for a cohort of $D$. suzukii

\begin{tabular}{|c|c|c|c|c|c|c|c|c|c|c|}
\hline Stage & $n$ & Interval $(x)$ & $l_{x}$ & $p_{x}$ & $q_{x}$ & $d_{x}$ & ${ }_{n} L_{x}$ & $T_{x}$ & $e_{x}$ & $c_{x}$ \\
\hline Egg & 1.384 & $0-1$ & 1.000 & 0.868 & 0.132 & 0.132 & 1.293 & 56.194 & 56.194 & 0.250 \\
\hline $\mathrm{Ll}$ & 1.059 & $1-2$ & 0.868 & 0.913 & 0.087 & 0.075 & 0.879 & 54.902 & 63.256 & 0.142 \\
\hline L2 & 1.477 & $2-3$ & 0.792 & 0.952 & 0.048 & 0.038 & 1.143 & 54.022 & 68.171 & 0.155 \\
\hline L3 & 3.115 & $3-6$ & 0.755 & 0.900 & 0.100 & 0.075 & 2.233 & 52.880 & 70.066 & 0.211 \\
\hline Pupa & 5.836 & $6-12$ & 0.679 & 0.944 & 0.056 & 0.038 & 3.854 & 50.646 & 74.563 & 0.163 \\
\hline $\mathrm{Al}$ & 10 & $12-22$ & 0.642 & 1.000 & 0 & 0 & 6.415 & 46.792 & 72.941 & 0.065 \\
\hline $\mathrm{A} 2$ & 10 & $22-32$ & 0.642 & 1.000 & 0 & 0 & 6.415 & 40.378 & 62.941 & 0.011 \\
\hline A 3 & 10 & $32-42$ & 0.642 & 1.000 & 0 & 0 & 6.415 & 33.962 & 52.941 & 0.002 \\
\hline A4 & 10 & $42-52$ & 0.642 & 0.971 & 0.029 & 0.019 & 6.321 & 27.547 & 42.941 & \\
\hline A5 & 10 & $52-62$ & 0.623 & 0.727 & 0.273 & 0.170 & 5.378 & 21.226 & 34.091 & \\
\hline A 6 & 10 & $62-72$ & 0.453 & 0.875 & 0.125 & 0.057 & 4.245 & 15.849 & 35.000 & \\
\hline A7 & 10 & $72-82$ & 0.396 & 0.905 & 0.095 & 0.038 & 3.774 & 11.604 & 29.286 & \\
\hline A 8 & 10 & $82-92$ & 0.358 & 0.737 & 0.263 & 0.094 & 3.113 & 7.830 & 21.842 & \\
\hline A9 & 10 & $92-102$ & 0.264 & 0.714 & 0.286 & 0.075 & 2.264 & 4.717 & 17.857 & \\
\hline Al0 & 10 & $102-112$ & 0.189 & 0.400 & 0.600 & 0.113 & 1.321 & 2.453 & 13.000 & \\
\hline All & 10 & $112-122$ & 0.075 & 0.250 & 0.750 & 0.057 & 0.472 & 1.132 & 15.000 & \\
\hline A12 & 10 & $122-132$ & 0.019 & 1.000 & 0 & 0 & 0.189 & 0.660 & 35.000 & \\
\hline A13 & 10 & $132-142$ & 0.019 & 1.000 & 0 & 0 & 0.189 & 0.472 & 25.000 & \\
\hline A14 & 10 & $142-152$ & 0.019 & 1.000 & 0 & 0 & 0.189 & 0.283 & 15.000 & \\
\hline A15 & 10 & $152-162$ & 0.019 & 0 & 1.000 & 0.019 & 0.094 & 0.094 & 5.000 & \\
\hline A16 & 10 & $162-172$ & 0 & & & & & & & \\
\hline
\end{tabular}

L1, first instar; L2, second instar; L3, third instar; and the A stages represent consecutive adult intervals of $10 \mathrm{~d}$. $n$ is the number of days in the interval and interval $(x)$ is the age interval associated with each stage. All formulae were taken from Carey (1982, 1993). 
velopment between these hosts (Lee et al. 2011a, Bellamy et al. 2013), illustrating that host can affect the development of D. suzukii. The differences in egg production and longevity between the current study and Kanzawa's work could partly be attributable to the host studied. However, it is also plausible that in the Ontario ecotype adult lifespan has increased, thereby increasing average lifetime egg production.

Here, we documented a significant difference in the mean gross maternity for the nonreplacement male group and replacement male group, where the nonreplacement male group experienced a greater mean gross maternity. Firstly, there is the possibility that the act of replacing the males in the replacement male group actually caused some disruption within the mating chamber, resulting in a delay in mating. However, Drosophila females are able to store sperm within their seminal receptacle and spermathecae, and so the difference in gross maternity between the male groups may be due to sperm competition between consecutive males (Price et al. 1999). Evidence suggests that male Drosophila can physically dislodge and incapacitate sperm stored within the female (Price et al. 1999), and this may have caused decreased gross maternity in the replacement male group.

We also documented a significant difference in the mean hatch rate for the nonreplacement male group and replacement male group, where the replacement male group experienced greater overall hatch rate of eggs. This suggests that there may be a difference in the reproductive period of male and female D. suzukii. The lower mean hatch rate of the nonreplacement male group may be a consequence of males becoming infertile earlier than females (i.e., females have a longer reproductive period than males, and therefore are able to produce eggs longer than males are able to produce sperm). As females are still able to lay unfertilized eggs, we see gross maternity maintained but the hatch rate of these eggs decrease. This difference suggests a role for female choice behavior in field environments. A female who is able to mate with a fertile male would receive direct fitness benefits in the form of more viable eggs, resulting in higher offspring production (Droney 1996). Overall, it has been found that multiple matings in Drosophila increases female fitness (Arnqvist and Nilsson 2000). Although the replacement male group experienced a lower mean gross maternity, the higher hatch rate of this group may offset the lower egg production, leading to greater overall fitness.

The $M_{x}$ data suggest that egg production is maximized at intermediate ages (Fig. 1). However, for the nonreplacement male group and the replacement male group, we observe different peaks in egg production ( $\approx 70 \mathrm{~d}$ vs. $\approx 40 \mathrm{~d}$, respectively), as well as different best-fit curves. The difference in the ages of peak egg production could possibly be a result of the fact that males increase in quality as they mature. Klepsatel et al. (2013) describe four phases relating to D. melanogaster fecundity in a female's life: 1) reproductive maturation reaching peak fecundity within 3-4 d;2) prolonged linear decrease in fecundity; 3 ) exponential decrease in fecundity; and 4) postovipository period with no eggs laid until mortality. Excluding the initial peak in egg production, D. suzukii do not seem to reach their next peak in fecundity until much later in life (Fig. 1). After this period, we do see a decrease in fecundity; however, this decrease does not become exponential and there is very little, if any, postovipository life.

$H$, which is a measure of heterogeneity in the probability of dying at each age (Carey 1993) was found to be 0.683 . According to our life table (Table 4), $e_{x}$ increases when an individual reaches the pupal stage. This is caused by the fact that mortality at immature stages is high compared with adult mortality, and therefore when an individual has survived that life stage (i.e., the pupal stage), it is likely to survive longer than originally expected.

Longevity has been found to be highly dependent on temperature. When Partridge et al. (1995) investigated longevity of adult $D$. melanogaster, they reported a significant increase in longevity at $16^{\circ} \mathrm{C}$ as compared with $25^{\circ} \mathrm{C}$. Our study was conducted at $22^{\circ} \mathrm{C}$, which has been reported as the preferred temperature of D. suzukii (Calabria et al. 2010, Walsh et al. 2011). A temperature-dependent life table remains an important research goal for this important species.

The $r$ is a valuable parameter because it combines mortality and fecundity to yield an estimate of growth (Zahari et al. 2010). Our estimate (0.179) is similar to those found for other species of the melanogaster species group. For example, in Drosophila serratar Malloch averaged $\approx 0.115$ at $20^{\circ} \mathrm{C}$ and $\approx 0.211$ at $25^{\circ} \mathrm{C}$ (Birch et al. 1963). Birch et al. demonstrated that $r$ could vary with temperature and location. It is a reasonable assumption that $D$. suzukii's $r$ also depends on temperature, and again argues for the need to construct a temperature-dependent life table.

This study confirms the enormous potential of $D$. suzukii. In addition to being used as a starting point to parameterize population models, which can be used to predict characteristics such as number of generations per year and population trends, these baseline data provide some insights into the problem of pest monitoring and control for this species. It is clear that $D$. suzukii is a comparatively long-lived, fecund fruit fly with a growth rate allowing a population to double in size in as little as $4 \mathrm{~d}$. Our estimate of the stable age distribution shows that only $8 \%$ of the population comprises adults. An estimate similar to what has been found for other insects (Birch 1948). Our stable age distribution estimate can have implications for current monitoring methods. Presently, baited traps are used to monitor D. suzukii adults. Given that adults may only represent a small portion of the population, quantifying infestation based on trap counts may not be accurate, and perhaps these traps are only useful for determining the presence or absence of $D$. suzukii. The potential for explosive population growth, and the fact that adults appearing in traps probably comprise a very small fraction of the total population, both suggest that pest management strategies should be applied as soon as the pest appears in a field. 


\section{Acknowledgments}

We would like to thank Justin Renkema, Denise Beaton, and Hannah Fraser for fly samples; and Adam Cave for the fly rearing and laboratory diet protocol. Funding for this research came from grants from the Ontario Ministry of Agriculture and Food, and the Canadian Natural Science and Engineering Research Council, to J. N.

\section{References Cited}

Arnqvist, G., and T. Nilsson. 2000. The evolution of polyandry: multiple mating and female fitness in insects. Anim. Behav. 60: 145-164.

Beers, E. H., R. A. Van Steenwyk, P. W. Shearer, W. W. Coates, and J. A. Grant. 2011. Developing Drosophila suzukii management programs for sweet cherry in the western United States. Pest Manage. Sci. 67: 1386-1395.

Bellamy, D. E., M. S. Sisterton, S. S. Walse. 2013. Quantifying host potentials: Indexing postharvest fresh fruits for spotted wing drosophila, Drosophila suzukii. PLoS ONE 8: $1-10$.

Birch, L. C. 1948. The intrinsic rate of natural increase of an insect population. J. Anim. Ecol. 17: 15-26.

Birch, L. C., T. H. Dobzhansky, E. O. Elliot, and R. C. Lewontin. 1963. Relative fitness of geographic races of Drosophila serrata. Evolution 17: 72-83.

Bolda, M. P., R. E. Goodhue, and F. G. Zalom. 2010. Spotted wing drosophila: potential economic impact of a newly established pest. Agric. Resour. Econ. 13: 5-8.

Calabria, G., J. Maca, G. Bachli, L. Serra, and M. Pascual. 2010. First records of the potential pest species Drosophila suzukii (Diptera: Drosophilidae) in Europe. J. Appl. Entomol. 136: 139-147.

Carey, J. R. 1982. Demography and population dynamics of the Mediterranean fruit fly. Ecol. Modell. 16: 125-150.

Carey, J. R. 1993. Applied demography for biologists with special emphasis on insects. Oxford University Press, New York, NY.

(CFIA) Canadian Food Inspection Agency. 2011. RMD-1101: Pest risk management document - Drosophila suzukii (spotted wing drosophila). (http://www.inspection.gc.ca/ plants/plant-protection/directives/risk-management/ rmd-11-01/eng/1330738873775/1330738972893).

Cini, A., C. Ioriatti, and G. Anfora. 2012. A review of the invasion of Drosophila suzukii in Europe and a draft research agenda for integrated pest management. B. Insectol. 65: 149-160.

Dalton, D. T., V. M. Walton, P. W. Shearer, D. B. Walsh, J. Caprile, and R. Isaacs. 2011. Laboratory survival of Drosophila suzukii under simulated winter conditions of the Pacific Northwest and seasonal field trapping in five primary regions of small and stone fruit production in the United States. Pest Manage. Sci. 67: 1368-1374.

Droney, D. C. 1996. Environmental influences on male courtship and implications for female choice in a lekking Hawaiian Drosophila. Anim. Behav. 51: 821-830.

Hauser, M. 2011. A historic account of the invasion of Drosophila suzukii (Matsumura) (Diptera: Drosophilidae) in the continental United States, with remarks on their identification. Pest Manage. Sci. 67: 1352-1357.
Huey, R. B., G. W. Gilchrist, M. L. Carlson, D. Berrigan, and L. Serra. 2000. Rapid evolution of a geographic cline in size in an introduced fly. Science 287: 308-309.

Kanzawa, T. 1939. Studies on Drosophila suzukii Mats. Kofu. Rev. Appl. Entomol. 29: 622.

Klepsatel, P., M. Galikova, N. De Maio, S. Ricci, C. Schlotterer, and T. Flatt. 2013. Reproductive and post-reproductive life history of wild-caught Drosophila melanogaster under laboratory conditions. J. Evol. Biol. 26: 1508 1520 .

Lee, J. C., D. J. Bruck, H. Curry, D. Edwards, D. R. Haviland, R. A. Van Steenwyk, and B. M. Yorgey. 2011a. The susceptibility of small fruits and cherriesto the spotted-wing drosophila, Drosophila suzukii. Pest Manage. Sci. 67: 1358-1367.

Lee, J. C., D. J. Bruck, A. J. Dreves, C. Ioriatti, H. Vogt, and P. Baufeld. 2011b. In focus: spotted wing drosophila, Drosophila suzukii, across perspectives. Pest Manage. Sci. 67: 1349-1351.

Levitan, M., and W. J. Etges. 2005. Climate change and recent genetic flux in populations of Drosophila robusta. BMC Evol. Biol. 5: 1-11.

McMillan, I., M. Fitz-Earle, L. Butler, and D. S. Robson. 1969. Quantitative genetics of fertility II. Lifetime egg production of Drosophila melanogaster-experimental. Genetics 65: 355-369.

Partridge, L., B. Barrie, N. H. Barton, K. Fowler, and V. French. 1995. Rapid laboratory evolution of adult lifehistory traits in Drosophila melanogaster in response to temperature. Evolution 49: 538-544.

Polanco, A. M., C. C. Brewster, and D. M. Miller. 2011. Population growth potential of the Bed Bug, Cimex lectularius L.: a life table analysis. Insects 2: 173-185.

Price, C.S.C., K. A. Dyer, and J. A. Coyne. 1999. Sperm competition betweenDrosophila males involvesboth displacementand incapacitation. Nature 400: 449-452.

Rose, M. R., and B. Charlesworth. 1980. Genetics of life history in Drosophila melanogaster. II. Exploratory selection experiments. Genetics 97: 187-196.

SAS Institute. 2013. Using JMP 11. SAS Institute, Cary, NC.

Stenger, D. C., M. S. Sisterson, and R. French. 2010. Population genetics of Homalodisca vitripennis reovirus validates timing and limited introduction to California of its invasive insect host, the glassy-winged sharpshooter. Virology 407: 53-59.

Taylor, M. L., N. Wedell, and D. J. Hosken. 2008. Sexual selection and female fitness in Drosophila simulans. Behav. Ecol. Sociobiol. 62: 721-728.

Walsh, D., M. Bolda, R. Goodhue, A. J. Dreves, J. C. Lee, D. J. Bruck, V. M. Walton, S. D. O’Neal, and F. G. Zalom. 2011. Drosophila suzukii (Diptera: Drosophilidae): Invasive pest of ripening soft fruit expanding its geographic range and damage potential. Integ. Pest Manage. 106: 289-295.

Zahari, B., Y. Fathipour, M. Khanjani, S. Moharramipour, and M. P. Zalucki. 2010. Modeling demographic response to constant temperature in Hypera postica (Coleoptera: Curculionidae). J. Econ. Entomol. 103: 292-301.

Received 20 November 2013; accepted 22 April 2014. 